\title{
QPFORSETI
}

Forseti. Revista de Derecho. Volumen 11, No 15, Lima, 2022, pp. 92 - 116

\section{Los mejores esfuerzos (best efforts) en la contratación corporativa y financiera}

\author{
Sergio García Long*
}

\begin{abstract}
Resumen. - El presente trabajo trata sobre el uso de la cláusula de mejores esfuerzos (best efforts) en la práctica corporativa y financiera. Para ello, se desarrolla a los best efforts según la práctica americana e inglesa para luego analizar cómo dicha cláusula anglosajona puede ser entendida en general en jurisdicciones civilistas. Finalmente, se analiza en concreto a la cláusula hell or high water y al contrato de best efforts underwriting.

Abstract. - This paper deals with the use of best efforts clause in corporate and financial transactions. For this purpose, best efforts clause is explained according to American and English practice to later analyze how such Anglo-Saxon clause may be understood in general under civilian jurisdictions. Finally, hell or high water clause and best efforts underwriting contract are specifically analyzed.
\end{abstract}

Palabras claves. - Contratos - mejores esfuerzos - hell or high water - underwriting.

Keywords. - Contracts - best efforts - hell or high water - underwriting.

* Abogado por la Pontificia Universidad Católica del Perú (PUCP) con mención summa cum laude. Su actividad profesional y académica se enfoca en Derecho de Contratos, Societario, Fusiones \& Adquisiciones, Financiamientos y Arbitraje. Asociado en Vargas Pareja Abogados. Ha sido adjunto de docencia en los cursos Derecho de las Obligaciones, Responsabilidad Civil, Análisis Económico del Derecho y Temas de Derecho Societario en la PUCP. 


\section{Introducción}

La institución contractual de los best efforts (mejores esfuerzos) es ampliamente usada en la práctica contractual anglosajona. A pesar de tal origen, también es pactada en contratos sujetos a ley de jurisdicciones civilistas, como la peruana. La intención del presente trabajo es enfocarse en el estudio de los best efforts conforme al Common Law para luego explicar cómo puede ser entendida en una jurisdicción del Civil Law. Con ocasión de ello, se desarrollarán dos figuras contractuales usadas en la práctica corporativa y financiera: la cláusula hell or high water y el contrato de best efforts underwriting 1 .

Para tales efectos, la sección II explica el motivo por el cual es eficiente para las partes pactar cláusulas generales, ambiguas y vagas. La sección III se enfoca en el estudio de la cláusula de best efforts conforme al Common Law y cómo la misma debe ser entendida en el Civil Law. Luego, la sección IV desarrolla la cláusula hell or high water en las operaciones de M\&A, mientras que la sección V trata sobre el contrato de underwriting en operaciones financieras $y$, en específico, sobre la modalidad best efforts. La sección VI concluye.

\section{Contratos incompletos y vaguedad estratégica}

Una primera interrogante que surge cuando pensamos en términos contractuales vagos como los mejores esfuerzos, la buena fe, la equidad, los estándares aceptados por la industria, entre otros, es la siguiente: ¿por qué se pactan términos vagos y generales en vez de definir y redactar con precisión los comportamientos que deben ejecutar las partes? La respuesta está en el costo de obtener información y de las negociaciones.

Si las partes tuvieran los recursos necesarios (tiempo y dinero) para definir todas las posibles contingencias que puedan surgir en el curso de su relación contractual, posiblemente definirían todo tipo de evento adverso: desde declaraciones de guerra hasta la caída de un gran meteorito. Sin embargo, ello no toma en cuenta dos cuestiones fundamentales: (1) que es una suposición falsa que las partes tengan y estén dispuestas a gastar todos sus recursos en celebrar un contrato, y (2) que no tiene sentido económico regular un evento adverso cuando la probabilidad de ocurrencia del mismo es tan baja que incurrir en costos de negociación resulta un desperdicio.

Por lo mencionado, es deseable y sobre todo eficiente que las partes ahorren en costos de información y negociación y opten por celebrar un contrato incompleto

1 Previamente hemos explicado el pacto de la cláusula de best efforts para el derecho de contratos en general. En esta oportunidad nos enfocamos en dos supuestos específicos, la cláusula hell or high water en operaciones de fusiones y adquisiciones, y el best efforts underwriting en operaciones de financiamientos. Véase GARCÍA LONG, Sergio. “Lo que importa es la intención: la cláusula de mejores esfuerzos (best efforts)". Apuntes desde el derecho peruano hacia el derecho comparado. En Revista de Derecho - Facultad de Derecho UCSC, Vol. 39, 2021, p. 125-161. 
pero eficiente en lugar de un contrato completo pero ineficiente ${ }^{2}$. El diseño contractual que opten las partes debe tener en cuenta que no es responsabilidad de ellas regular todo tipo de comportamiento ex ante pues resulta conveniente que ciertas conductas sean resueltas ex post por un tercero en caso surja una controversia. Nuevamente, tiene sentido económico regular ciertas conductas, pero no otras.

Una primera aproximación se encuentra en la diferencia entre reglas (rules) y estándares (standards) ${ }^{3}$. Las reglas son aquellas disposiciones que se encuentran claramente definidas en su contenido, mientras que los estándares son lo opuesto al no presentar un contenido preciso. Una regla puede ser una cláusula de pago de una compraventa, el cual define el modo y tiempo del pago del precio (cuantía, tipo de moneda, cuenta bancaria, cronograma de pagos, entre otros). Por el contrario, un estándar puede consistir en cumplir una obligación de buena fe o realizar los mejores esfuerzos para intentar conseguir un resultado. La eficiencia se encuentra en el trade off entre los costos de negociación frente a los costos de cumplimiento y verificación.

Por definición la regla involucra altos costos de negociación, pero bajos costos de

2 HART, Oliver y MOORE, John. Incomplete Contracts and Renegotiation. En Econometrica, núm. 4, 1988, p. 755: «The difficult task facing the drafters of a contract is to anticipate and deal appropriately with the many contingencies which may arise during the course of their trading relationship. Since it may be prohibitively costly to specify, in a way that can be enforced, the precise actions that each party should take in every conceivable eventuality, the parties are in practice likely to end up writing a highly incomplete contract»; HART, Oliver D. "Incomplete Contracts and the Theory of the Firm". En Journal of Law, Economics, E Organization, vol. 4, Núm. 1, 1988, p. 123: «[...] in practice, transaction costs are pervasive and large. A consequence of the presence of such costs is that the parties to a relationship will not write a contract that anticipates all the events that may occur and the various actions that are appropriate in these events. Rather they will write a contract that is incomplete, in the sense that it contains gaps or missing provisions [...]»; Posner, Eric A. Economic Analysis of Contract Law After Three Decades: Success or Failure? En The Yale Law Journal, Vol. 112, 2003, p. 833: «A theoretically complete contract would describe all the possible contingencies, but transaction costs -including the cost of negotiating and writing down the terms- and foreseeing low-probability events, render all contracts incomplete»; Baker, Scott y Krawiec, Kimberly D. Incomplete Contracts in a Complete Contract World. En Florida State University Law Review, Vol. 33, 2006, p. 731: «In the legal model, contracts are "obligationally incomplete" because, whether deliberately or by accident, contracting parties fail to fully specify at the outset of their relationship all of their rights and obligations under the contract».

3 KAPLOW, Louis. “Rules versus Standards: An Economic Analysis”. En Duke Law Journal, Vol. 42, 1992, p. 559-560: «Arguments about and definitions of rules and standards commonly emphasize the distinction between whether the law is given content ex ante or ex post. For example, a rule may entail an advance determination of what conduct is permissible, leaving only factual issues for the adjudicator: (A rule might prohibit "driving in excess of 55 miles per hour on expressways.") A standard may entail leaving both specification of what conduct is permissible and factual issues for the adjudicator. (A standard might prohibit "driving at an excessive speed on expressways")». Véase también Ehrlich, Isaac y Posner, Richard A. An Economic Analysis of Legal Rulemaking. En The Journal of Legal Studies, Vol. 3, Núm. 1, 1974; Diver, Colin S. The Optimal Precision of Administrative Rules. En The Yale Law Journal, Vol. 93, Núm. 65, 1993; Schäfer, Hans-Bernd. Legal rules and standards. En Rowley, Charles K. y Schneider, Friedrich (eds). The Encyclopedia of Public Choice. Kluwer New York, Academic Publishers, 2004; Schäfer, Hans-Bernd. Rules versus standards in rich and poor countries: precise legal norms as substitutes for human capital in low-income countries. En Supreme Court Economic Review, Vol. 14, 2006. 
cumplimiento y verificación. En nuestro ejemplo de la cláusula de pago de la compraventa, las partes tienen que invertir recursos en definir el tiempo y modo del pago desde que hay certeza que el comprador deberá pagar el precio. Por ello, tiene sentido gastar en redactar una cláusula precisa que sea una regla. Esta asunción de altos costos se compensa con los bajos costos de cumplimiento y verificación. Para que el comprador pueda cumplir con su obligación de pago del precio deberá seguir al pie de la letra lo que señala la cláusula respectiva. De esta manera, no debe asumir demasiados costos para saber cómo cumplir con su obligación. A su vez, en caso surja alguna controversia respecto al pago del precio del contrato, las partes podrán informar a los tribunales y, a bajo costo, lo sucedido en los hechos y sobre los derechos y obligaciones pactados. Cualquier tribunal podrá verificar con facilidad -leyendo el contrato y verificando los hechos- si el comprador cumplió o no. Si el comprador pagó el precio según el modo y tiempo pactado, el tribunal podrá verificar y concluir que el comprador no se encuentra en incumplimiento.

Por el contrario, el pacto de un estándar ${ }^{4}$-por definición- permite ahorrar en costos de negociación a cambio de un incremento en los costos de cumplimiento y verificación. Por ejemplo, pensemos en la adquisición de una empresa. Es común que las partes firmen el contrato de adquisición (constituyendo un contrato válido) pero que difieran la eficacia y/o exigibilidad del mismo hasta que no se cumpla con determinadas condiciones precedentes. Solo con el cumplimiento de las condiciones se procederá con el cierre del contrato (esto es, el contrato generará todos sus efectos principales).

La preocupación respecto al cierre surge cuando existen condiciones que no dependen exclusivamente del control de una de las partes. Por ejemplo, el comprador puede haber condicionado el cierre de la operación a la obtención de un financiamiento (que usará para pagar el precio). Claramente la obtención del financiamiento no depende exclusivamente del comprador dado que el préstamo está sujeto a la evaluación que realizará el banco respecto del comprador y la operación que pretende financiar. En estos casos, el vendedor se suele proteger incluyendo una cláusula por el cual le exige al comprador realizar sus mejores esfuerzos para obtener el financiamiento.

Si el vendedor realmente quisiera asegurarse que el banco aprobará el desembolso de los fondos, negociaría un listado de todas las conductas que el comprador deberá realizar frente al banco para obtener la aprobación deseada. Desde que para el vendedor es complicado pensar ex ante en todo lo que debería hacer el comprador para obtener el financiamiento y, sobre todo, lograr la incorporación en el contrato de todos aquellos comportamientos específicos (desde que es seguro que el comprador se negará aceptar tales términos), resulta más barato y

4 Sobre los estándares de fuente legal sobre el comportamiento del hombre, véase MEIER, José Francisco y HARMAN, Diego A. “Los estándares de hombre en el derecho: ¿qué tan alto debemos poner la valla jurídica?" En Revisa de Derecho y Economía, vol. 7, núm. 26, 2010. 
conveniente para avanzar las negociaciones que el vendedor se contente con incluir una cláusula de mejores esfuerzos.

Si el vendedor no incluye tal cláusula podría terminar obstaculizando las negociones y en el peor de los casos producir un deal-breaker. Dichas complicaciones se solucionan incluyendo un estándar a bajo costo como los mejores esfuerzos. Los bajos costos de negociación que implican pactar un estándar se justifican en los mayores costos de cumplimiento y verificación.

¿Cómo cumple el comprador con su obligación de realizar los mejores esfuerzos para obtener el financiamiento? El comprador solo sabe que debe realizar sus mejores esfuerzos, pero no sabe cómo cumplir con tal requerimiento. Entonces, será costoso para él probar que efectivamente cumplió. En caso de desacuerdo y controversia, las partes deberán acudir a las cortes. Pero las cortes se encuentran en una posición más complicada. Son las partes las que han celebrado y ejecutado el contrato $\mathrm{y}$, por ende, las protagonistas de tal relación contractual. Por el contrario, las cortes son extrañas a las partes y al contrato, y solo sabrán lo que pasó en los hechos en virtud de la evidencia que las partes provean al caso. Desde que el estándar no se encuentra definido, será costoso para las partes presentar la información que permita sustentar sus posiciones.

Incluso, esta distinción entre reglas y estándares puede servir para definir la técnica del legislador frente a la elaboración de normas legales. Si una jurisdicción tiene buenos jueces, el legislador podrá usar estándares (se confía que los jueces completarán correctamente el contenido de la norma general), pero si no es el caso, será mejor redactar reglas (a malos jueces, mejor implementar normas claras y precisas) $)^{5}$.

En resumen, las reglas son eficientes porque implican asumir mayores costos de negociación a cambio de menores costos de cumplimiento y verificación, mientras que los estándares son eficientes porque permiten ahorrar en costos de negociación a cambio de mayores costos de cumplimiento y verificación. Las reglas y estándares y sus respectivos costos también son explicados como términos precisos (precise terms) y términos vagos (vague terms). Como explican Scott y Triantis, el diseño contractual optado por las partes debe consistir en una combinación de términos contractuales precisos y términos contractuales vagos, en virtud del trade-off entre costos ex ante (front-end transaction costs) o costos ex post (back-end enforcement costs) 6 . Dado que el contrato completo no es económicamente eficiente, tiene sentido que en ciertas circunstancias las partes asuman altos costos de redacción, mientras que en otros casos es deseable que las

5 SCHÄFER, Hans-Bernd. "Rules versus standards in rich and poor countries: precise legal norms as substitutes for human capital in low-income countries". En Supreme Court Economic Review, Vol. 14, 2006.

6 SCOTT, Robert E. y TRIANTIS, George G. “Anticipating Litigation in Contract Design”. En The Yale Law Journal, vol. 115, 2006. 
partes asuman bajos costos de redacción y no definan un comportamiento debido y trasladen a los tribunales la tarea de definir tal comportamiento ex post.

Esto es precisamente lo que se hace cuando se pacta un estándar como los mejores esfuerzos ${ }^{7}$. Intencionalmente las partes se ponen de acuerdo en que no están de acuerdo y prefieren pactar una cláusula vaga en vez de definir un listado de comportamientos específicos. En nuestro ejemplo de la adquisición de empresa, las partes prefieren simplemente indicar que el comprador deberá realizar sus mejores esfuerzos para lograr el cierre del contrato. Así evitan asumir altos costos de redacción.

Este pacto de la cláusula de mejores esfuerzos -como el de cualquier otra cláusula general- es una habilitación expresa en favor de los tribunales para que sean éstos los que resuelvan, en caso de surgir alguna controversia, si el comprador ejecutó las conductas necesarias que permitan concluir si efectivamente realizó sus mejores esfuerzos.

Pero tengamos en cuenta que esta delegación en favor de los tribunales parte de la premisa de que los mismos podrán completar eficientemente en nombre de las partes lo que ellas no quisieron negociar en su momento. Sin embargo, debemos tener en cuenta que existen otros costos diferentes a los costos de ejecución del contrato: (1) la incertidumbre y (2) el error judicial.

Por definición, un estándar permite que las partes no se pongan de acuerdo respecto al mismo, si se ejecutó debidamente o no. Mientras que el vendedor señalará que el comprador no realizó sus mejores esfuerzos, el comprador se defenderá señalando que realizó todo lo que estuvo a su alcance. Esto aumenta las probabilidades de que las partes terminen en litigio $y$, si estamos ante un contrato de una cuantía importante, como son las adquisiciones de empresas, un financiamiento o una gran construcción, entonces las partes tendrán que asumir los altos costos de un litigio complicado. A ello debemos agregar la incertidumbre respecto a la decisión que pueda tomar el tribunal y el eventual error.

En efecto, si son las partes las protagonistas de su relación contractual y el tribunal un extraño, existe un claro supuesto de asimetría de información. Pero esta asimetría de la información no solo significa que las partes tengan más información sobre el caso concreto a diferencia del tribunal, sino -y más importante- significa que el tribunal deberá reconstruir lo que efectivamente ocurrió en los hechos en base a información tergiversada por las propias partes.

El demandante usará la información que tienen disponible y la presentará en el juicio de tal manera que pueda defender sus pretensiones, y lo mismo con el

7 ESPOSITO, Grant y KAUFMAN, Jessica. “'Best Efforts', 'Commercially Reasonable' And Other Terms No One Understands". En New York Law Journal, Commercial Litigation, 14 de marzo de 2016: «In business disputes, by contrast, we delegate the role of defining a broken boundary object to a third party -a court, a jury, or an arbitrator». 
demandado. Esta situación genera incertidumbre entre las partes respecto a cuál será el fallo del tribunal y crea el escenario perfecto para que los tribunales incurran en error judicial al considerar como hechos aquellos que en realidad nunca ocurrieron. Si los hechos que retiene el tribunal no son los reales, entonces el tribunal no elegirá correctamente la regla de derecho que debe usar para resolver el caso en concreto.

En virtud de tales consideraciones, se podría pensar que en realidad resulta eficiente pactar cláusulas precisas y no cláusulas vagas. Sin embargo, ello no se evidencia en la práctica desde que el uso de cláusulas vagas es común en operaciones corporativas y financieras. Términos vagos como mejores esfuerzos (best efforts), buena fe (good faith), razonabilidad (reasonableness), estándares aceptados por la industria (accepted industry standards), debida diligencia (due diligence), entre otros, se encuentran estandarizados en la práctica nacional e internacional ${ }^{8}$. Por ende, no podría señalarse sin mayor análisis que las cláusulas contractuales vagas son ineficientes 9 .

Un aspecto adicional que nos permite explicar por qué las cláusulas vagas son eficientes es que las partes las pactan siendo conscientes del inicio de un eventual litigio. El pacto de una cláusula contractual vaga tiene en cuenta la probabilidad de que una de las partes demande y los costos que ello involucra. A pesar de ello, y aunque pueda parecer contra intuitivo a primera vista, los altos costos de litigio, la incertidumbre respecto al pronunciamiento del tribunal y el error judicial, generan los incentivos para que las partes revoquen la habilitación otorgada a los tribunales para completar la cláusula vaga y sean finalmente ellas mismas quienes completen la cláusula que en un principio no quisieron completar.

Si los costos de litigio son muy altos y la incertidumbre y el error también, entonces se generan los incentivos entre las partes para que renegocien los términos del contrato. Así, las partes evitan asumir los altos costos de litigio y evitan la incertidumbre y el error al resolver ellas mismas la cláusula vaga ${ }^{10}$. Por el contrario, si tales costos son bajos, las partes seguirán con su plan inicial y dejarán que las cortes completen la cláusula.

La práctica corporativa ha confirmado el uso de cláusulas vagas y los incentivos

8 FONTAINE, Marcel. Best Efforts, Reasonable Care, Due Diligence et Règles de L'Art dans les Contrats Internationaux. En RDAI, núm. 8, 1988, p. 1003-1004.

9 TRIANTIS, George G. "The Efficiency of Vague Contract Terms: A Response to The SchwartzScott Theory of U.C.C." Article 2. En Louisiana Law Review, vol. 62, núm. 4, 2002, p. 1071: «A number of explanations have been offered for the decision of parties not to exploit some verifiable factors. First, the ex-ante transaction costs of specifying each possible verifiable future state of the world -even if they can all be foreseen and contemplated-may exceed the gains. The parties may have more cost-effective means at their disposal to address remote contingencies than legal enforcement of complete contract terms».

10 HILL, Claire A. Bargaining in the Shadow of the Lawsuit: A Social Norm Theory of Incomplete Contracts. En Delaware Journal of Corporate Law, Vol. 34, 2009, p. 208: «[...] the uncertainty and costs of litigation serve as a bond the parties give against precipitous recourse to litigation, aligning the parties' incentives to resolve any disputes without formal resort to the court system». 
que las mismas generan para renegociar el contrato. Por ejemplo, en los contratos de M\&A se suele pactar una cláusula MAC (Material Adverse Change) o MAE (Material Adverse Effect) en favor del comprador, la cual le permite terminar la operación sin pago de compensación en caso algo malo ocurra que deteriore el valor del target antes de la fecha de cierre ${ }^{11}$. En la práctica americana, el MAC ha sido alegado no necesariamente para dejar sin efecto el contrato sino para coaccionar a la otra parte a renegociar los términos contractuales, bajo apercibimiento de dejar sin efecto la operación. El comprador tiene el derecho a terminar el contrato, pero para que tenga éxito debe probar que efectivamente ocurrió un MAC, lo cual es complicado pues el MAC se pacta como una cláusula general que no contiene una definición precisa de lo que es un MAC. Ante este escenario, si el comprador tiene éxito podrá terminar la operación sin necesidad de pagar una compensación, pero si se equivoca, tendrá que cerrar la compra y pagar daños. Por el contrario, el vendedor se defenderá señalando que no ocurrió un MAC, pero tendrá el mismo problema que el comprador para probar la no ocurrencia de un MAC pues la misma cláusula no define qué es un MAC. Ante este escenario, si el vendedor tiene éxito el comprador deberá cerrar la operación y pagarle daños, pero si efectivamente ocurrió un MAC, se quedará sin operación y compensación. Dada la situación de las partes, ellas prefieren renegociar, y en efecto, muchas partes han renegociado el contrato ante la alegación de un MAC (por ejemplo, reduciendo el precio). En otras palabras, la alegación del MAC es más una amenaza que una realidad ${ }^{12}$.

Resumiendo lo señalado, la renegociación dependerá de qué tan altos sean los costos ex post: (1) las partes pactan una cláusula vaga porque la misma permite ahorrar en costos ex ante a cambio de mayores costos ex post. Si los costos ex post no son altos en relación al ahorro en costos ex ante, entonces las partes esperarán que un tribunal complete la cláusula vaga, por el contrario (2) si los costos ex post terminan siendo muy altos de manera que no se justifica el ahorro en los costos ex ante, entonces no tendrá sentido asumir mayores costos para que un tribunal resuelva la controversia, sino que será preferible que las mismas partes completen la cláusula vaga, esto es, procedan con una renegociación eficiente.

\section{La cláusula de best efforts}

11 En operaciones de M\&A el MAC y MAE son usados de manera indistinta (ambos enfocados en cambios o efectos adversos que afectan finalmente al target). Por otro lado, en financiamientos el MAC se refiere a riesgos sistémicos (cambios adversos) (por ello también suele ser denominado como Market MAC) mientras que el MAE sobre afectaciones al deudor, documentos del financiamiento, garantías y derechos del banco (efectos adversos).

12 GARCÍA LONG, Sergio. Un Big MAC, por favor: La cláusula MAC en fusiones y adquisiciones. Lima, Fondo Editorial PUCP, 2016, p. 210-211. La Corte de Delaware intentó dar algunas pautas sobre cómo interpretar el MAC y creó el estándar supletorio del comprador razonable a largo plazo. Este estándar fue uno alto y así se evidenció en diversos casos en donde no se amparó la ocurrencia de un MAC, como en IBP $v$. Tyson Foods (2001), Frontier Oil $v$. Holly Corporation (2005) y Huntsman $v$. Hexion (2008). Si bien en Akorn v. Fresenius (2018) la Corte de Delaware por primera vez reconoció la ocurrencia de un MAC, recientemente en Bardy Diagnostics v. HillRom (2021) se volvió a rechazar la ocurrencia de un MAC. 


\section{i. ¿Qué es la cláusula de best efforts?}

En el derecho comparado existen dos regímenes de responsabilidad contractual. En el Civil Law la responsabilidad contractual es generalmente subjetiva, de manera que el deudor debe incurrir en culpa para responder, caso contrario, no responderá si prueba la ausencia de culpa. Si se da el caso, también podría probar la ocurrencia de un impedimento (caso fortuito o fuerza mayor). En otras palabras, podría alegar dos defensas. Por el contrario, en el Common Law la responsabilidad contractual es generalmente objetiva, de manera que el deudor siempre responde, incluso si la obligación se volvía en imposible por la ocurrencia de un impedimento, como se indicó en el caso inglés Paradine v. Jane (1647). Posteriormente, desde Taylor v. Caldwell (1863) el derecho inglés admite la doctrina de la frustration como excusa al cumplimiento contractual. Para un civilista, los casos de frustration son supuestos específicos de imposibilidad. Entonces, una parte anglosajona responde a menos que pruebe la ocurrencia de un impedimento. En otras palabras, solo puede alegar una defensa. El régimen de responsabilidad objetiva también se encuentra en los principales instrumentos internacionales de uniformización de derecho de contratos como la CISG ${ }^{13}$, la cual solo admite la exoneración de responsabilidad contractual ante la ocurrencia de un impedimento conforme a su artículo 79.

Partiendo de la premisa que la responsabilidad contractual es objetiva, los common lawyers empezaron a pactar cláusulas específicas para limitar su responsabilidad. Ante la ausencia de pacto, respondían de manera objetiva. Por el contrario, si incorporaban algún pacto para aligerar el compromiso que asumían frente a la otra parte, tendrían mayores posibilidades de eximirse de responsabilidad. Así surgen las cláusulas de best efforts. La parte anglosajona no asume un compromiso absoluto frente a la otra, sino solo se compromete a realizar sus mejores esfuerzos para lograr un resultado sin garantizar la obtención del mismo. De esta manera, el énfasis deja de estar en el resultado para enfocarse en los esfuerzos, o diligencia como lo entenderían los civilistas.

En la estructura contractual de la cláusula de mejores esfuerzos se puede observar lo siguiente: (1) las partes identifican el resultado deseado, (2) una parte se compromete a realizar sus mejores esfuerzos para lograr la obtención del resultado sin definir cuáles serían los comportamientos idóneos para conseguir tal resultado, y (3) no se garantiza la obtención del resultado.

Según lo anterior, dos aspectos se mantienen como inciertos: (a) las conductas idóneas que son objeto de los mejores esfuerzos, y (b) la obtención del resultado deseado.

Es importante prestar atención a cómo las partes pueden definir el nivel de diligencia para intentar lograr un resultado sin necesidad de garantizar la

13 Acrónimo de United Nations Convention on Contracts for the International Sales of Goods. 
obtención del resultado. Por ejemplo, si no se pacta algo particular en el contrato, un prestador de servicios común deberá ejecutar su obligación ejerciendo la diligencia ordinaria, que es el estándar de diligencia que se aplica de manera supletoria ${ }^{14}$. Pero las partes podrían pactar niveles de diligencia superior como "los estándares de la industria", "el comerciante ordenado" o cualquier otro. Aunque no se garantiza un resultado, al menos se exige a una parte a tratar más o poner mayor esfuerzo, sin definir aún qué comportamiento específico puede ser idóneo para aumentar las posibilidades de obtener el resultado. Pero al menos se sabe que hay que intentar más.

Por eso la cláusula de mejores esfuerzos es una cláusula general, imprecisa, vaga e indefinida. ¿Por qué las partes pactaría una cláusula de este tipo? Hay situaciones en donde las partes no tienen la información suficiente para definir ex ante todo lo que tiene que hacer una parte para lograr un resultado. Entonces, resulta eficiente solo señalar que una parte deberá realizar sus mejores esfuerzos, y así se evita incluir en el contrato un listado de comportamientos específicos que una parte deberá realizar. Esto permite ahorrar en costos de transacción. Si posteriormente surge una controversia en torno a los comportamientos que debía realizar una parte en virtud de la cláusula de mejores esfuerzos para saber si realmente realizó -vale la redundancia- sus mejores esfuerzos, entonces las partes tendrán que negociar nuevamente para definir una solución, caso contrario, intervendrán las cortes. En este segundo momento las partes asumen ex post los costos que ahorraron ex ante cuando optaron por pactar una cláusula general, imprecisa, vaga e indefinida.

Ahora, el uso de diferentes estándares puede estar enfocado en la diligencia como también en calidad o cantidad del resultado. Por ejemplo, un artista se puede obligar a pintar un cuadro, cuya calidad recién se podrá apreciar una vez terminado el cuadro. La calidad no será la misma si el cuadro será usado en una casa privada que a ser exhibida en un museo. Dentro de este escenario, existe un resultado comprometido, pero aun así queda indefinida la calidad de tal resultado. Igualmente, un distribuidor se compromete a vender bienes, y realizará sus mejores esfuerzos para vender la mayor cantidad de bienes. Aunque se compromete a un resultado (vender) aún queda como contingente la cantidad de los bienes vendidos, lo cual dependerá directamente de cuánto se haya esforzado.

Si bien la cláusula de mejores esfuerzos está enfocada inicialmente en la diligencia (donde no se garantiza un resultado), también puede afectar la calidad o cantidad del resultado comprometido (donde sí se garantiza un resultado). En efecto, en la práctica se suele usar a los mejores esfuerzos para calificar el resultado, cuya calidad o cuantía es inicialmente indefinida y dependerá del ejercicio de los mejores esfuerzos, pero partiendo de la premisa que hay un resultado mínimo

14 En Perú, conforme al artículo 1314 del Código Civil. En caso de prestaciones de especial dificultad, aplicará el estándar del artículo 1672 del Código Civil. 
comprometido. Por ello, será necesario verificar el pacto para concluir cuál es el verdadero ámbito de aplicación de los mejores esfuerzos.

Los interesante de la cláusula de mejores esfuerzos es que, aunque en teoría no define nada ex ante, los common lawyers han propuesto diferentes técnicas de redacción (drafting) para intentar definir lo indefinido sin llegar a una definición absoluta. En la práctica contractual se han definido diferentes niveles de esfuerzos que debe realizar una parte sin llegar a definir un comportamiento específico. Aunque no se fija un comportamiento fijo, al menos -dentro de la misma indefinición- se emplean diferentes niveles de exigencias de esfuerzos o diligencia. No es lo mismo pactar que una parte deberá realizar sus "esfuerzos razonables" que sus "máximos esfuerzos". Así, es posible pactar diversos niveles de esfuerzos. La referencia a una "cláusula de mejores esfuerzos" es solo una mención general. Es necesario revisar el pacto para verificar qué nivel específico de esfuerzos se pactó en el caso concreto.

\section{ii. El drafting anlosajón}

El Common Law parte de una perspectiva formalista y textualista respecto a la interpretación de los contratos, a diferencia del contextualismo del Civil Law. El juez anglosajón es muy respetuoso del pacto de las partes y de la aplicación de reglas legales o convencionales que limiten la interpretación del contrato a sus cuatro esquinas. Por ello, existen reglas como el parol evidence rule (no se admite evidencia extrínseca para complementar la interpretación del contrato), plain meaning rule (si no hay duda se debe preferir por el significado ordinario de las palabras), merger clause (el acuerdo total de las partes está en lo redactado en el contrato) y non-oral modification clause (el contrato solo se modifica por escrito). Por el contrario, en el Civil Law se prefiere por una interpretación contextualista que tenga en cuenta las circunstancias del caso, el comportamiento de las partes antes, durante y después del contrato, y las exigencias de la buena fe.

Además, en el Common Law -sobre todo por el derecho inglés- se maneja una posición anti-buena fe, mientras que en el Civil Law la buena fe es un concepto ampliamente aceptado. Incluso, se reconoce la función integrativa de la buena fe $\mathrm{e}^{15}$. Bajo este escenario, si existe la buena fe como cláusula general con el poder de integrar el contrato, las partes civilistas pueden redactar contratos menos extensos, a diferencia de los anglosajones que redactan contratos más largos porque parten del textualismo y el rechazo de usar cláusulas generales ${ }^{16}$.

15 Sobre los diferentes roles de la buena fe en el derecho comparado, véase García Long, Sergio. La muerte de la buena fe: reflexiones en torno al rol de la buena fe en el derecho comparado. Primera Parte. En Actualidad Civil, Núm. 37, julio 2017; García Long, Sergio. La muerte de la buena fe: reflexiones en torno al rol de la buena fe en el derecho comparado. Segunda Parte. En Actualidad Civil, Núm. 38, agosto 2017.

16 HILL, Claire A. y KING, Christopher. How do German contracts do as much with fewer words? En Chicago-Kent Law Review, Vol. 79, No. 3, 2004; García Long, Sergio. El nacimiento y ascenso de los civil lawyers. En Themis - Revista de Derecho, No. 73, 2018; García Long, Sergio. La 
Partiendo de estas premisas, los americanos no manejan una sola cláusula de mejores esfuerzos sino diversas cláusulas según el estándar específico que se pacte. En la práctica americana existe una jerarquía de esfuerzos: (1) best efforts, (2) reasonable best efforts, (3) reasonable efforts, (4) commercially reasonable efforts, y (5) good faith efforts ${ }^{17}$. Con el objetivo de lograr una precisión perfecta, se ha hecho uso de una semántica excesiva18. Esta clasificación debe ser vista de manera ilustrativa. Mientras que los abogados han definido esta jerarquía, a veces las cortes no han respetado la misma y la han alterado al interpretarlas de manera distinta ${ }^{19}$.

En primer lugar, el best efforts es el estándar más riguroso y oneroso. "Best" significa hacer todo lo posible para lograr el resultado, incluso asumir un costo excesivo y sacrificar los propios intereses en favor de la otra parte. Si bien en principio los abogados consideraban que el best efforts necesariamente imponía un estándar gravoso, con el tiempo las cortes señalaron que dicha cláusula debía cumplirse dentro de parámetros de razonabilidad, de manera que una parte puede tomar en cuenta sus intereses y no está obligada a hacer todo lo posible incluso en perjuicio propio ${ }^{20}$. En segundo lugar, tenemos al reasonable best efforts. Este sería un estándar menor al best efforts. Se añade el "reasonable" antes del "best" para matizar el estándar más exigente. El tercer lugar, el reasonable efforts reduce aún más el nivel de exigencia pues reemplaza el "best" por el "reasonable". En cuarto lugar, tenemos al commercially reasonable efforts. Mientras que algunos consideran que la inclusión del "commercially" adelante del "reasonable efforts" tiene el efecto de reducir el nivel de esfuerzos exigido, otros consideran que lo aumenta. También hay quienes señalan que no habría diferencia entre el "commercially reasonable efforts" y el "reasonable efforts". Finalmente, el "good faith efforts" puede considerarse como el nivel menos exigente desde que "good faith" solo implica

americanización del derecho de contratos. En Velasco Valderas, Patricia Jannett (ed). Derecho Corporativo. Alcances y Perspectivas. Lima: Be Lawyer, 2021.

17 Sobre los mejores esfuerzos en el derecho americano, véase Goetz, Charles J. y Scott, Robert E. Principles of Relational Contracts. En Virginia Law Review, Vol. 67, Núm. 6, 1981; Adams, Kenneth. Understanding “Best Efforts” And Its Variants (Including Drafting Recommendations). En The Practical Lawyer, Agosto 2004; Sergent, Randolph Stuart. Do Your Best with "Best Efforts": Using Open Contract Terms. En Maryland Bar Journal, Marzo 2007; Fox, Charles M. Working with Contracts. Second Edition. New York, Practising Law Institute, 2008; Adams, Kenneth. Delaware "Efforts" Caselaw and Why It's Irrelevant. En Adams on Contract Drafting, 21 de octubre, 2013.

18 ADAMS, Kenneth. Beyond Words. En Solicitors Journal, Vol. 158, Núm. 37, 2014, p. 21; Arnold \& Porter. Delaware Court Addresses the Meaning of "Commercially Reasonable Efforts" in M\&A Context. En Arnold \& Porter Advisory, 29 de junio 2016.

19 HILL, Claire A. y King, Christopher. How do German contracts do as much with fewer words? En Chicago-Kent Law Review, Vol. 79, No. 3, 2004; García Long, Sergio. El nacimiento y ascenso de los civil lawyers. En Themis - Revista de Derecho, No. 73, 2018; García Long, Sergio. La americanización del derecho de contratos. En Velasco Valderas, Patricia Jannett (ed). Derecho Corporativo. Alcances y Perspectivas. Lima: Be Lawyer, 2021.

20 Véase el leading case americano Bloor $v$. Falstaff (1979). Incluso, algunos autores consideran que es mejor hablar de "reasonable efforts" y no de "best efforts" (para eliminar el best). Véase Adams, Kenneth. With 'efforts' provisions, reasonable is better than best. En The Lawyers Weekly, Business Law, 16 de mayo 2014. 
buenas intenciones en este contexto (recordemos que la buena fe americana no es un concepto fuerte como la buena fe civilista alemana, por ejemplo).

En la práctica contractual inglesa se reemplaza el uso del término "efforts" por "endeavours", aunque ambos son sinónimos y pueden ser traducidos al castellano como "esfuerzos". A los americanos les gustan "efforts" mientras que a los ingleses "endeavours", quienes también cuentan -en principio- con una jerarquía de endeavours: (1) best endeavours, (2) all reasonable endeavours, y (3) reasonable endeavours ${ }^{21}$. Al igual que en la experiencia americana, esta jerarquía debe ser tomada como ilustrativa pues las cortes han interpretado esta jerarquía de manera distinta ${ }^{22}$.

En primer lugar, "best endeavours" impone el estándar más oneroso por el "best" pero dentro de lo razonable. De hecho, en su momento se hizo famosa la frase " $[t o]$ leave no stone unturned" (no dejar ni una piedra sin mover) conforme al caso Sheffield District Railway v. Great Central Railway (1911)23, uno de los más antiguos y leading case inglés. Sin embargo, al igual que en el derecho americano, actualmente en el derecho inglés se considera que el best endeavours no impone un nivel de diligencia ilimitado sino uno razonable24. En segundo lugar, el "all reasonable endeavours" impone un nivel de exigencia menor, mientras que en tercer lugar se encuentra el reasonable endeavours. Una novedad en la técnica del drafting inglés es el uso del "all". Mientras que "all reasonable endeavours" imponer realizar "todos" los actos posibles (sin ser tan exigentes como el "best endeavours"), el "reasonable endeavours" solo implica realizar un solo curso de acción al no tener el "all".

\section{iii. El estándar de cumplimiento}

Señalábamos que la cláusula de mejores esfuerzos debe entenderse como un género desde que en la práctica se pueden pactar diversos niveles de esfuerzos como se muestra en la experiencia americana e inglesa. De esta manera, aunque no se define qué comportamientos específicos son debidos y no se garantiza el resultado deseado, al menos se logra distinguir entre niveles de esfuerzos. Esto permite que se pueda usar una cláusula general y ambigua, pero a su vez, lograr cierta precisión.

Por ello, dentro de esta imprecisión aún quedaba pendiente una gran interrogante, ¿cómo sabe una parte cuándo ha cumplido con sus mejores esfuerzos? La doctrina

21 Sobre los mejores esfuerzos en el derecho inglés, véase Young, Jessica. An endeavour to understand endeavours undertakings. En Hong Kong Law Journal, Vol. 44, Núm. 1, 2014; Yi, Tan Tian. The interpretation of endeavours clauses. En Singapore Academy of Law Journal, Vol. 27, Núm. 1, 2015.

22 Véase el caso Rodhia International Holdings Ltd. v. Huntsman International (2007).

23 FONTAINE, Marcel. Best Efforts, Reasonable Care, Due Diligence et Règles de L'Art dans les Contrats Internationaux. En RDAI, Núm. 8, 1988, p. 1006; Young, Jessica. An endeavour to understand endeavours undertakings. En Hong Kong Law Journal, Vol. 44, Núm. 1, 2014, p. 97.

24 Véase los casos Terrel v. Mabie (1952) y Malik Co v. Central European Trading Agency (1974). 
americana propuso diversos estándares de cumplimiento a ser tomados en cuenta: good faith (según la buena fe), diligence insurance (priorizar el interés del deudor), fiduciary duty (priorizar el interés del acreedor), joint maximization (maximizar el interés de ambas partes), subjective (tomar en cuenta la particular posición del deudor y sus habilidades), objetive (aplicar un estándar general como el hombre prudente, el hombre razonable, el buen padre de familia, independiente de la posición del deudor), entre otros. Por el lado de la jurisprudencia, no existe una guía clara al respecto 25 .

Estos estándares de cumplimiento son empleados al momento que el deudor debe elegir qué conducta ejecutar para intentar lograr el resultado. Ciertamente habrá conductas más idóneas que otras. Nuevamente, aunque no se defina alguna conducta específica, al menos se puede aplicar alguna guía para que el deudor sepa cómo elegir bien en favor del acreedor. Por ejemplo, el estándar del fiduciary duty implica que el deudor (agente) subordine su interés en favor del acreedor (principal), mientras que el joint maximization toma en cuenta el interés de ambas partes. No se elegirá el mismo curso de acción si el deudor sabe que debe subordinar su interés, a que no tenga que hacerlo.

$\mathrm{Al}$ respecto, debemos comentar lo señalado en el caso LTV Aerospace v. Thomson (1996), en donde se indicó que "A party may thus exercise discretion, within its good faith business judgment, in devising a strategy for achieving its ultimate goal"26. Esto significa que la elección de las conductas idóneas para pretender el resultado debe realizarse según la buena fe, pero no cualquier buena fe sino la good faith business judgment que hace referencia al derecho corporativo.

Los administradores de una sociedad están sujetos al cumplimiento de deberes fiduciarios, entre ellos, el deber de diligencia (duty of care) y el deber de lealtad (duty of loyalty $)^{27}$. Dado que los mercados son inciertos y que en determinadas ocasiones es necesario tomar decisiones riesgosas, en el derecho corporativo se creó un límite a la responsabilidad de los administradores según el business judgment rule28, por el cual, si el administrador tomó una decisión informada y

25 Véase Miller, Zachary. Best efforts?: Differing judicial interpretations of a familiar term. En Arizona Law Review, Vol. 48, Núm. 3, 2006; Park, Rob. Putting the "best" in best efforts. En University of Chicago Law Review, Vol. 73, Núm. 2, 2006.

26 LTV Aerospace and Defense Co. v. Thomson (In re Chateaugay), 198 B.R. 848 (S.D.N.Y. 1996), p. 854.

27 En Perú, véase el artículo 171 de la Ley General de Sociedades, y los artículos 147 y 148 del Anteproyecto de Ley General de Sociedades (2018).

28 WACHTELL, Lipton, Rosen \& Katz. Takeover Law and Practice. NY, Wachtell, Lipton, Rosen \& Katz, 2016, p. 28: «[...] the business judgment rule is a presumption that directors are complying with their fiduciary duties. The purpose of the rule is to "encourage corporate fiduciaries to attempt to increase stockholder wealth by engaging in those risks that, in their business judgment, are in the best interest of the corporation 'without the debilitating fear that they will be held personally liable if the company experiences losses" ».

Wachtell, Lipton, Rosen \& Katz (2016). Takeover Law and Practice (NY, Wachtell, Lipton, Rosen \& Katz). 
diligente, no será responsable en caso ocurra algún daño en perjuicio de la empresa.

Actualmente, y en relación a los deberes fiduciarios, también se discute sobre el duty of good faith que deben cumplir los administradores y su relación con el business judgment rule, lo que ha dado como resultado que se hable de un good faith business judgment ${ }^{29}$. Esto significa que los administradores, cuando tengan que tomar una decisión, deberán emplear la buena fe al momento de elegir entre todas las alternativas.

El empleo de la buena fe, como estándar de cumplimiento, es ilustrativo para entender cómo puede funcionar cualquier estándar de cumplimiento en favor del acreedor. Si el deudor no tiene ningún parámetro, entonces elegirá por cualquier tipo de comportamiento, incluso aquellos que no sean idóneos para conseguir el resultado. Por el contrario, si el deudor tiene alguna guía ello le permitirá saber cuándo ha cumplido con su compromiso aunque no haya logrado el resultado, mientras que a la misma vez se protegerá al acreedor en caso el deudor ejecute comportamientos inútiles.

Desde que en jurisdicciones civilistas la buena fe suele estar reconocida por ley y como un estándar imperativo ${ }^{30}$, puede considerarse como el estándar mínimo que aplicará a todo deudor que deberá realizar sus mejores esfuerzos, salvo que se pacte un estándar mayor.

\section{iv. ¿Cómo se debe entender la cláusula de best efforts en el Civil Law?}

Habiendo explicado cómo funciona la cláusula de mejores esfuerzos en el Common Law, se observa que la misma se enfoca en la diligencia, pero no solo en ella, sino en definir diversos estándares de diligencia. Este punto es clave para diferenciar a los efforts de su equivalente civilista, las obligaciones de medios (obligations de moyens) ${ }^{31}$. Usualmente se considera que la cláusula de mejores esfuerzos es solo un pacto para incluir una obligación de medios, de manera que su inclusión en un contrato sujeto a ley civilista sería redundante en el sentido que no significaría la importación de una figura anglosajona a la respectiva jurisdicción

29 Véase EISENBERG, Melvin A. The Duty of Good Faith in Corporate Law. En Delaware Journal of Corporate Law, Vol. 31, 2006; Strine, Leo E. Jr.; Hamermesh, Lawrence A.; Balotti, Franklin R. y Gorris, Jeffrey M. Loyalty's Core Demand: The Defining Role of Good Faith in Corporation Law. En The Georgetown Law Journal, Vol. 98, 2010; Griffith, Sean J. Good Faith Business Judgment: A Theory of Rhetoric in Corporate Law Jurisprudence. En Duke Law Journal, Vol. 55, Núm. 1, 2005.

30 En Perú, véase los artículos 168 y 1362 del Código Civil.

31 FARNSWORTH, E. Allan. On Trying to Keep One's Promises: The Duty of Best Efforts in Contract Law. En University of Pittsburgh Law Review, Vol. 46, 1984, p. 3; Pejovic, Caslav. Civil Law and Common Law: Two Different Paths Leading to the Same Goals. En Victoria University of Wellington Law Review, Vol. 32, Núm. 3, 2001, p. 826; Arbesú Gonzáles, Vanesa. La responsabilidad civil en el ámbito de la cirugía estética. Madrid, Dykinson, 2016, p. 44-45; Acevedo Rehbein, Alberto. Los mejores esfuerzos (best efforts) en el derecho contemporáneo de los contratos. En Revista de Estudios Socio-Jurídicos, Vol. 8, Núm. 2, julio-diciembre 2006, p. 117; Witz, Claude y Bopp, Thomas M. "Best Efforts, Reasonable Care: Considerations de Droit Allemand. En RDAI, Núm. 8, 1988, p. 1035-1037). 
civilista $^{32}$. Si bien esto puede ser cierto, es incompleto. La obligación de medios implica ejecutar la prestación conforme a la diligencia ordinaria (el estándar supletorio), mientras que una cláusula de mejores esfuerzos puede incorporar un nivel de diligencia superior a la diligencia ordinaria, lo cual significa un agravamiento de la responsabilidad contractual. Entonces, mientras que en algunos casos la cláusula de mejores esfuerzos será igual a una obligación de medios, en otros será algo distinto: un pacto que eleva el nivel de diligencia sin obligarse a obtener un resultado específico o, en otras palabras, una extensión de la responsabilidad contractual.

Otro aspecto importante que distingue a los best efforts de las obligations de moyens es que el objeto de la segunda normalmente está determinado, mientras que en la primera no existe precisión alguna. El deudor solo se obliga a realizar sus mejores esfuerzos, sin mayor precisión; por el contrario, en una obligación de medios el deudor sabe qué deberá hacer. Esto es así desde que los best efforts son una cláusula general de fuente convencional, y como tal, no debe estar delimitada a tal nivel como ocurre con las obligaciones, cuya prestación debe cumplir con el requisito de determinación.

Igualmente, los mejores esfuerzos son distintos a la buena fe. Si bien ambos pueden ser entendidos como estándares, los mejores esfuerzos significan la incorporación de un estándar más exigente. Para empezar, la buena fe ya se encuentra reconocida por ley en las jurisdicciones del Civil Law. Entonces, si se incorpora una cláusula de mejores esfuerzos, la misma no puede interpretarse como una redundancia sino como algo distinto que las partes quisieron incorporar en el contrato, caso contrario, la cláusula no tendría ninguna utilidad práctica. Desde que los mejores esfuerzos no tienen como fuente la ley, se debe partir de la premisa que su pacto significa incluir algo distinto a los estándares ya definidos por ley.

En resumen, para darle valor a la cláusula de mejores esfuerzos, no puede considerarse que su incorporación en el contrato significa otra forma de referirse a las obligaciones de medios o a la buena fe. La cláusula de mejores esfuerzos significa que una parte debe cumplir con un estándar mayor a los ya señalados por ley, y como tal, su pacto significa una modificación convencional de la responsabilidad contractual a efectos de agravarla o extenderla. Esta cláusula de mejores esfuerzos podría materializarse en cualquier nivel de diligencia según las jerarquías creadas por la práctica americana e inglesa.

Las precisiones realizadas previamente son esenciales para comprender que la cláusula de mejores esfuerzos puede usarse en el Civil Law de dos maneras: (1)

32 Por ejemplo, el artículo 5.1.4 de los PICC (UNIDROIT Principles of International Commercial Contracts) en su versión en inglés se refiere al best efforts mientras que en su versión en francés a las obligations de moyens. Esto podría llevarnos a pensar que se tratan de sinónimos. Sin embargo, si bien esto puede ser así en ciertas ocasiones, la práctica contractual demuestra que la aplicación de los best efforts en más compleja, y como tal, puede exceder al ámbito de aplicación de las obligations de moyens. 
como cláusula general de fuente convencional, y (2) como calificadores (qualifiers) de obligaciones.

En primer lugar, el Civil Law ya cuenta con la buena fe como cláusula general de fuente legal. Adicionalmente, la práctica contractual ha creado cláusulas generales como la MAC/MAE o los best efforts. La ventaja de estas cláusulas es que las partes no deben definir ex ante el contenido desde que el mismo se determinará ex post cuando sea aplicada. Entonces, en lugar que las partes tengan que definir un listado de conductas para lograr un resultado deseado, bastará pactar que se deberá realizar los mejores esfuerzos.

En segundo lugar, los best efforts pueden funcionar como calificador de obligaciones. El uso de calificadores es usual en la contratación corporativa y financiera para amplia o extender el contenido de una obligación. Si uno pacta una obligación de medios sin mayor mención, entonces aplicará la diligencia ordinaria. Por el contrario, una obligación de medios podría estar calificada por un nivel de diligencia mayor al ordinario a través de una cláusula de mejores esfuerzos.

Sin embargo, tal vez lo más interesante es observar cómo los best efforts podrían calificar también a una obligación de resultados, lo cual pone en evidencia por qué los best efforts no son sinónimos de las obligations de moyens. Aunque el resultado esté garantizado, podría quedar como contingente la calidad o cantidad del resultado (recordemos el ejemplo del artista o el distribuidor). En tales casos estamos ante obligaciones de resultados, y aunque el resultado esté garantizado no se sabe cuál será la calidad o cantidad del mismo. Así es como las obligaciones de resultados también podrían estar calificados por una cláusula de mejores esfuerzos, en donde está comprometido un resultado mínimo y el resultado final se conocerá una vez realizado los mejores esfuerzos.

En virtud de lo anterior, será necesario revisar el pacto para definir ante qué escenario nos encontramos. No es lo mismo pactar una obligación de medios con mejores esfuerzos que una obligación de resultado con mejores esfuerzos. Si un deudor se obliga solo a una obligación de medios pero calificada con mejores esfuerzos, no responderá si no logra obtener el resultado, pero sí será responsable si no cumple con desplegar el mayor esfuerzo requerido por pacto. Igualmente, si un deudor se obliga a obtener un resultado pero calificado con mejores esfuerzos, será responsable aunque obtenga un resultado siempre que tal resultado no sea aquél que se hubiera obtenido de haber desplegado el mayor esfuerzo requerido.

\section{Hell, or high water clause}

En operaciones de M\&A existe una cláusula de mejores esfuerzos con esteroides. Nos referimos a la cláusula "hell or high water" $(\mathrm{HHW})^{33}$. Dicha cláusula se

33 LYONS, Peter; Buffier, Beau; Fort, Tamara y Jennejohn, Matthew. Reasonable Best Efforts: Cold Comfort to Sellers. En The M\&A Lawyer, Vol. 18, Núm. 1, 2014, p. 7: «The gold-standard of covenants in this context is the so-called "hell or high water" (HOHW) provision, which requires 
entiende por su propio nombre que se deriva de la expresión americana "come hell or high water", que se usa para hacer referencia a la obtención de un resultado sin importar lo que se tenga que hacer incluso si en el camino se sufre consecuencias adversas. En castellano ello equivale a decir que el comprador debe "mover mar y tierra" o enfrentarse "contra viento y marea" para conseguir el resultado.

Por ello, en virtud de una cláusula HHW una parte debe realizar todo lo que sea necesario sin importa el costo involucrado y las dificultades que puedan presentarse, a efectos de lograr el resultado deseado. Este tipo de pacto es comúnmente usado en la práctica americana cuando el comprador debe obtener algo de un tercero para poder cerrar la operación, en específico, la autorización gubernamental por parte de la autoridad que vela por la libre competencia (antitrust) o la que regula determinado sector de la economía (por ejemplo, el bancario).

En una operación de M\&A suele distinguirse entre el signing (firma) y closing (cierre) del contrato. La firma del contrato significa que ya existe un contrato válido pero cuya eficacia está supeditada al cumplimiento de determinadas condiciones precedentes (conditions precedent), las cuales pueden tener como objeto obligaciones (covenants), declaraciones y garantías (reps \& warranties), cláusula $\mathrm{MAC} / \mathrm{MAE}$, entre otros. El cierre del contrato significa el momento en que el contrato produce todos sus efectos, entre ellos, la transferencia de la empresa y el pago del precio ${ }^{34}$. Pero el cierre solo ocurrirá si se cumplen con todas las condiciones precedentes pactadas. Mientras que hay condiciones que se encuentran dentro del ámbito de control de alguna de las partes, hay otras que dependen de acciones de terceros, entre ellas, la aprobación sobre libre competencia o aspectos regulatorios. En estos casos, las partes pactan como condición la obtención del comprador de la respectiva aprobación gubernamental. Esto significa que el riesgo es asumido por el vendedor pues en caso no se obtenga la aprobación se habrá incumplido una condición y el comprador podrá terminar el contrato.

Sin embargo, si bien la aprobación depende de un tercero, el comprador no puede tener una actitud pasiva frente a la autoridad. Se prefiere por una actitud activa que consista en realizar las diligencias necesarias para obtener el resultado esperado. Además, las autoridades suelen solicitar la realización de ciertas prestaciones o requerimientos antes de otorgar la respectiva aprobación, las cuales pueden representar la asunción de costos importantes. Dentro de este contexto, es

that the buyer must to whatever it takes to obtain regulatory approval, including agreeing to make divestitures or take any other actions necessary for approval».

34 Sobre la estructura contractual de los contratos de M\&A, véase Fox, Charles M. Working with Contracts. Second Edition. New York, Practising Law Institute, 2008; Coates IV, John C. M\&A Contracts: Purposes, Types, Regulation, and Patterns of Practice. En The European Corporate Governance Institute, Working Papers Series in Law, Law Working Paper, No. 292, 2015; Adams, Kenneth. The structure of M\&A contracts. USA: Legalworks, 2016; García Long, Sergio. Un Big MAC, por favor: La cláusula MAC en fusiones y adquisiciones. Lima, Fondo Editorial PUCP, 2016; Escobar Rozas, Freddy. Contratación corporativa y evolución legal. En Themis, No. 70, 2016. 
interés de las partes asignar este costo. Ante ello, las partes pueden pactar una cláusula HHW para exigir que el comprador realice "whatever is necessary" 35 (todo lo que sea necesario), sin importar el costo o las exigencias que requiera la autoridad para aprobar de la operación ${ }^{36}$. Si se pacta un HHW y el comprador se rehúsa a cumplir un requerimiento de la autoridad, será responsable por incumplimiento contractual por más oneroso que resulte tal requerimiento. Así, las partes asignan el antitrust risk o el riesgo regulatorio, pero sobre todo su respectivo costo.

Como explica Harkrider en relación al antitrust risk:

"Merger parties frequently allocate antitrust risk through contractual provisions in the merger agreement. At one extreme, these risk-shifting contractual provisions allow the buyer to walk away from the contract if the antitrust agency issues a second request or threatens to challenge the deal. At the other extreme, these provisions require the buyer to agree to any divestiture or other remedy that the antitrust agency demands -in other words, to close the deal "come hell or high water"" 37.

Al final, lo que pretende la cláusula HHW es exigirle al comprador que asuma cualquier costo económico que sea necesario para obtener la aprobación gubernamental que permita perfeccionar la adquisición. Así, la cláusula HHW se pacta en favor del vendedor. En todo caso, el comprador podría pactar alguna excepción a la cláusula HHW para que tenga alguna salida en su favor en caso la autoridad requiera algo irrazonable (por ejemplo, se podría pactar que el comprador deberá realizar todo lo necesario para cerrar la operación salvo que el costo de ello supere cierto monto dinerario).

Si bien la cláusula HHW puede asimilarse en su funcionalidad a los mejores esfuerzos, la primera es más gravosa que la segunda ${ }^{38}$ desde que los mejores esfuerzos deben ejecutarse de manera razonable, el cual es un límite que no se aplica a la cláusula HHW. La intención de esta última es que el comprador haga lo

35 COATES IV, John C. M\&A Contracts: Purposes, Types, Regulation, and Patterns of Practice. En The European Corporate Governance Institute, Working Papers Series in Law, Law Working Paper, No. 292, 2015, p. 11.

36 Van de Voorde, Mark; Pupkin, Barry A. y Chelberg, Stephen E. Sharing Regulatory Risk in Global M\&A. En Association of Corporate Counsel, Diciembre 2015, p. 30: «[...] there are several other provisions that can be used to apportion risks between parties. Probably the most onerous of these strategies involves the use of a hell-or-high-water clause. Such a clause requires the buyer to accept remedies required by a regulatory authority as a precondition for the parties to close a transaction. The buyer obligates itself to do whatever is necessary, come "hell or high water," to close the deal. The clause typically requires the buyer to accept any remedial action imposed by a government regulator, which is required as a precondition of closing».

37 HARKRIDER, John D. Risk-Shifting Provisions and Antitrust Risk: An Empirical Examination. En Antitrust, American Bar Association Section of Antitrust Law, Fall 2005, p. 52.

38 TUCKER, Darren S. y Yingling, Kevin L. Keeping the Engagement Ring: Apportioning Antitrust Risk with Reverse Break up Fees. En Antitrust, Vol. 22, Núm. 3, 2008, p. 72: «The most extreme form of a "best efforts" clause is a so called hell or high water clause. This provision mandates that, "come hell or high water," the buyer accept any remedial action required by a governmental authority to consummate the deal. This infrequently used obligation completely shifts the antitrust risk to the buyer». 
que sea para obtener la aprobación de la autoridad competente, esto es, "come hell or high water".

Un caso real resulta útil para graficar las diferencias entre ambas cláusulas y comprender cómo funciona la cláusula HHW en las adquisiciones de empresas. La firma de private equity Blackstone tenía la intención de comprar Alliance Data System, Inc. (ADS) por \$ 7.5 billones a través de un LBO (leveraged buyout o compra apalancada) ${ }^{39}$. ADS era el proveedor más grande de tarjetas de crédito. La Office of the Comptroller of the Currency (OCC) rechazó otorgar la aprobación regulatoria para que ADS sea adquirida por Blackstone debido a que el nivel de deuda que adquiriría ADS la dejaría sub-capitalizada. La OCC señaló, sin embargo, que aprobaría la transacción si Blackstone otorgara una garantía de $\$ 400$ millones en favor de los pasivos bancarios de ADS. Como podía esperarse, Blackstone se negó a desembolsar tal cantidad de dinero.

ADS demandó a Blackstone ante la Delaware Chancery Court para que otorgara la garantía. Como Blackstone es una firma de private equity que adquiere activos a través de LBO's, la estructura corporativa de la transacción era particular. La firma de private equity o el fondo respectivo nunca firma el contrato. Siempre se constituye al menos una SPV (Special Purpose Vehicle) o shell corporation, esto es, una subsidiaria cuyo propósito es ser la parte contractual que adquiere al target, y por ende, no tiene mayores activos que el dinero necesario para pagar el precio de la compra, y posteriormente, las mismas acciones del target que adquirión ${ }^{40}$. El objetivo de la firma de private equity es mantenerse alejado de la operación para mitigar riesgos.

Dado que Blackstone no era parte del contrato, solo se comprometió a realizar el aporte de capital necesario (equity commitment letter) en favor de la SPV para que pague el precio ${ }^{41}$ y otorgar una garantía en caso la subsidiaria decida cancelar la operación pagando un monto dinerario (reverse termination fee) ${ }^{42}$. Pero en ningún momento firmó el contrato de adquisición y, en consecuencia, ADS no tenía alguna acción contractual contra Blackstone al ser este un tercero.

39 DAVIDOFF, Steven M. Gods at War. Shotgun Takeovers, Government by Deal, and the Private Equity Implosion. New Jersey, Wiley, 2010, p. 85-87.

40 Sobre la estructura contractual y corporativa del LBO, véase García Long, Sergio. Mega transacciones: la estructura contractual y corporativa del leveraged buyout (LBO). En Salazar Gallegos, Max (ed). Derecho Corporativo. Estudios en Homenaje a la Facultad de Derecho PUCP en su Centenario. Lima, PUCP, 2019.

41 Cuando el comprador es un SPV, los vendedores requieren cierta garantía por parte de los sponsors (en este caso, la firma de private equity), para que el SPV proceda a pagar el precio en el tiempo y modo acordado si se cumplen todas las condiciones precedentes. Como se sabe que la SPV no tiene activos y que el dinero vendrá de los sponsors, estos suelen emitir equity commitment letter, con o sin carácter vinculante, para asegurar al vendedor que si se cumplen todas las condiciones precedentes, se procederá a capitalizar al SPV para que se cancele el precio.

42 El reverse termination fee es el derecho de salida pactado en operaciones de M\&A. En virtud de este derecho, el comprador puede terminar la operación, sin necesidad de aviso previo y de manera discrecional, siempre que proceda a pagar el fee pactado (como compensación en favor de la otra parte). 
Teniendo en cuenta esta estructura contractual y corporativa, ADS quiso defender la siguiente tesis en una demanda judicial: el contrato exigía a la SPV realizar sus reasonable best efforts para obtener cualquier aprobación regulatoria, entre ellas, la de la OCC. Entonces, en virtud de sus reasonable best efforts, la SPV tenían que exigir, incluso judicialmente, a su matriz Blackstone para que desembolsara la garantía solicitada por la OCC.

En primer lugar, en Delaware no existe aún un completo entendimiento sobre la institución de los best efforts y lo que se requiere según cada tipo de efforts, en este caso, el reasonable best efforts. En segundo lugar, como la SPV era una subsidiaria que no tenía activos propios, cualquier demanda que presentara ADS contra la SPV no tenía utilidad si la SPV no tenía activos para responder (por ejemplo, por breach of contract y compensatory damages). En tercer lugar, el camino optado por ADS, incluso si fuera exitoso, tenía complicaciones prácticas. ADS tenía que ganar la demanda contra la SPV para que una corte declarase que los reasonable best efforts obligan a la SPV a exigir a Blackstone el desembolso de la garantía solicitada por la OCC, pero esto no aseguraba que efectivamente Blackstone fuera a desembolsar la garantía (seguramente Blackstone alegaría alguna defensa frente a la SPV para negarse a ello). Finalmente, ADS retiró la demanda y el contrato se dejó sin efecto luego que se cumpliera el drop-deadline.

Este caso no solo demuestra lo favorable que es la estructura corporativa de una adquisición liderada por una firma de private equity a través de un LBO, sino que es un buen ejemplo que demuestra la diferente utilidad de la cláusula HHW frente a los best efforts. ADS hubiera estado en una mejor posición si hubiera pactado un HHW, desde que tal cláusula sí exigiría a la SPV a requerir, incluso judicialmente, a su matriz el desembolso de la garantía solicitada por la autoridad, hasta que consiga el efectivo desembolso, sin importar lo que sea necesario (come hell or high water). A diferencia de los mejores esfuerzos, el HHW no está sujeto a la razonabilidad.

\section{El contrato de best efforts underwriting}

En el ámbito bancario y financiero tenemos al contrato de underwriting ("underwrite" puede traducirse al castellano como "garantizar" o "suscribir"). El contrato de underwriting es firmado entre un banco de inversión (a veces también llamado banco estructurador $\mathrm{y} / \mathrm{o}$ colocador) y una empresa emisora de instrumentos de deuda. Debido a que la emisora no tiene experiencia en vender deuda al público, o a privados, necesita de un agente que le ayude a colocar dicha deuda. En virtud de un contrato de underwriting el banco (o underwriter) asume en favor del emisor cierto compromiso en relación a la colocación de la deuda, a efectos que el underwriter asuma -en diferentes grados- el riesgo de una colocación no exitosa. La naturaleza del contrato de underwriting no es una sola desde que el 
objeto del mismo puede consistir en un aseguramiento y/o colocación, lo cual dependerá de las tres modalidades de underwriting generalmente conocidas ${ }^{43}$.

Primero, en virtud del firm commitment underwriting (o underwriting en firme), el banco compra toda la deuda por parte del emisor y la revende posteriormente en el mercado. En esta modalidad el banco asume todo el riesgo de colocación. Este tipo de underwriting es muy favorable para el emisor dado que le asegura todo el capital que pretendía levantar. Así, en esta modalidad de underwriting hay aseguramiento más servicio de colocación. Incluso algunos autores consideran que, en realidad, un firm commitment underwriting no es una especie de aseguramiento sino un contrato de suscripción de valores ${ }^{44}$, cuyo precio es uno especial para que el underwriter pueda ganar un diferencial con la reventa.

Segundo, en virtud del standby underwriting, el banco se compromete adquirir todos los valores que no se logren vender después de la colocación. En este caso, el underwriter no asegura la totalidad del riesgo de colocación sino solo parte de ella, y una vez conocida la demanda. En esta modalidad de underwriting también es beneficiosa para el emisor desde que hay aseguramiento más servicio de colocación, aunque un aseguramiento en menor grado.

Tercero, la modalidad más favorable para el underwriter es el best efforts underwriting45, por el cual el banco solo se compromete a realizar sus mejores esfuerzos para colocar la mayor cantidad de valores en el mercado, sin obligarse a comprar la deuda o garantizar los valores que no puedan venderse. De esta manera, el riesgo lo asume el propio emisor pues el banco no asegura la colocación, solo realiza un servicio. Entonces no hay aseguramiento, solo servicio de colocación. Esta modalidad funciona como una verdadera intermediación a diferencia de los otros supuestos de underwriting en donde está presente un elemento de aseguramiento. Por este motivo, en la experiencia americana se ha cuestionado que el best efforts underwriting sea una modalidad de underwriting si

43 Sobre la naturaleza del contrato de underwriting, véase Vidal Ramírez, Fernando. La bolsa de valores. Un ensayo de derecho bursátil. Lima: Cultural Cuzco, 1988; Thorndike Piedra, Thomas. El contrato de underwriting: El aseguramiento de ofertas públicas de valores mobiliarios. En Tratado de Derecho Mercantil. Tomo III. Contratos Mercantiles y Bancarios. Lima: Gaceta Jurídica, 2008; Avendaño, Jorge. El contrato de underwriting. En Contratos Civiles y Modernos de Empresa. Volumen II. Lima: MFC Editores, 2010; García Long, Sergio. La estructura contractual de los financiamientos bancarios. En García Long, Sergio (ed). Derecho Contractual Financiero. Estudios en Homenaje a la Facultad de Derecho PUCP en su Centenario. Lima, PUCP, 2019.

44 S.H. The Nature and Function of Underwriting Agreements. En University of Pennsylvania Law Review and American Law Register, Vol. 79, Núm. 7, 1931, p. 942.

45 CZEPIEL, Lori Anne. Best Efforts Underwriting: Does Glass Steagall Allow it? En Annual Review of Banking Law, vol. 7, 1988, p. 563-564. 
no existe aseguramiento de por medio46. Igualmente, desde una perspectiva civil se considera que el best efforts underwriting sería un mandato mercantil ${ }^{47}$.

El contrato de best efforts underwriting nos permite evidenciar la extensión que puede tener la institución contractual de los best efforts: no solo puede constituir un pacto contractual sino también puede dar lugar a un contrato típico y nominado. El best efforts underwriting, además, nos permite evidenciar cómo los mejores esfuerzos no se limitan a calificar el nivel de diligencia sino también el resultado esperado. Como hemos indicado, esto representa una de las principales novedades que puede tener los best efforts en jurisdicciones del Civil Law, los cuales suelen ser asimilados a las obligaciones de medios (obligations de moyens). Sin embargo, el ámbito de aplicación de los best efforts es mayor, y como tal, también pueden aplicarse a las obligaciones de resultado, de manera que se garantiza un resultado, pero queda incierto el aspecto cuantitativo o cualitativo del resultado.

En la práctica se suele discutir si es necesario que en el contrato de best efforts underwriting se pacte expresamente el tipo de obligación que asume el banco frente al emisor, esto es, si se trata de una obligación de medios y no. Pensamos que existe esta discusión porque se cree que existe una sinonimia perfecta entre las obligaciones de medios y los best efforts, lo cual no es correcto pues reduce el gran ámbito de aplicación que tienen los best efforts como se observa en la práctica contractual anglosajona.

En nuestra opinión, no se puede decir que en el best efforts underwriting no se garantiza ningún resultado pues es evidente que el emisor contrata a un especialista financiero para que coloque -efectivamente- instrumentos de deuda. Se espera un resultado mínimo en relación a la totalidad de la deuda. Lo que queda como contingente es la cantidad de valores que serán vendidos (bonos, por ejemplo), y dicha cantidad dependerá de los mayores o menores esfuerzos que realice el banco en la ejecución del contrato. Mientras más se esfuerce el banco, más bonos venderá. Así, los mejores esfuerzos se centran en el nivel de diligencia, pero a efectos de conseguir el mejor resultado posible, partiendo de la premisa que se está asegurando un resultado mínimo. Por ello, entiéndase que en virtud de un best efforts underwriting se asume una obligación de resultado, no una de medios. El underwriter no puede señalar que cumple el contrato incluso si no vende ningún bono. Desde que se contrata a un agente especializado, su trabajo implica un éxito mínimo. Lo que se discutirá, en todo caso, será es si el éxito mínimo es conforme al nivel de diligencia o esfuerzos pactado en el contrato.

46 Véase S.H. The Nature and Function of Underwriting Agreements. En University of Pennsylvania Law Review and American Law Register, Vol. 79, Núm. 7, 1931; Mandelker, Gershon, \& Raviv, Arthur. Investment banking: an economic analysis of optimal underwriting contracts. En The Journal of Finance, Vol. 32, No. 3, 1977.

47 Vidal Ramírez, Fernando. La bolsa de valores. Un ensayo de derecho bursátil. Lima: Cultural Cuzco, 1988, p. 233. 
Entonces, mientras que en una cláusula de best efforts el resultado no está garantizado, en el best efforts underwriting un resultado mínimo es garantizado porque se contrata a un agente financiero con experiencia. Si el banco no coloca ningún instrumento de deuda será claro que incumplió con realizar sus mejores esfuerzos. No podría señalar que solo se comprometió a realizar sus mejores esfuerzos sin garantizar ningún resultado.

Lo anterior debe considerarse como el punto de partida pues siempre será posible el pacto en contra, el cual debe ser respetado. Si las partes pactan expresamente que en virtud del contrato de best efforts underwriting se asume una obligación de medios, entonces así será. Pero si el contrato no dice nada al respecto, debe entenderse que se trata de una obligación de resultado, cuyo resultado está calificado por mejores esfuerzos (según el nivel de diligencia pactado) de manera que está garantizado un resultado mínimo, pero queda como contingente el elemento cuantitativo y/o cualitativo del resultado, según corresponda.

Fíjese que esta interpretación sobre la naturaleza del best efforts underwriting no agrava la posición de banco si tenemos en cuenta que en estos contratos se pactan condiciones precedentes a la colocación. Entre estas condiciones están la ausencia de un MAE (que nada malo le pase al emisor) o la ausencia de un MAC o Market MAC (que nada malo ocurra en los mercados financieros, la economía, la política u cualquier otro riesgo sistémico $)^{48}$. Por ejemplo, si se produce un cambio adverso en los mercados financieros que impide que el banco pueda colocar exitosamente la deuda, podrá suspender el contrato sin responsabilidad mientras se haya incumplido la condición, e incluso -si así fue pactado- podría terminar el contrato. Pero si nada malo ha ocurrido, el banco -como agente financiero especializadono tiene excusa en caso no pueda colocar ningún instrumento de deuda. Su experiencia le exige lograr un resultado mínimo, caso contrario, estará en incumplimiento contractual frente al emisor.

\section{Reflexiones finales}

Entre las reglas fundamentales del derecho de contratos se encuentra la libertad de elegir con quién contratar y la libertad de determinar el contenido del contrato. Este último significa que las reglas sobre contratos, en su mayoría, son reglas dispositivas y supletorias. Las normas imperativas son la excepción. Dentro este contexto, la ley de contratos (el Código Civil) es solo el punto de partida desde que las partes siempre podrán derogarla por pacto.

48 Sobre el MAC en operaciones financieras, véase Molina Rey de Castro, Fernando. Las cláusulas MAC en financiamientos y adquisiciones. En Freddy Escobar \& Cecilia O'Neill (eds). Ensayos de derecho contractual financiero. Lima, Fondo Editorial de la Universidad del Pacífico, 2013; Manzanares, Alberto. Una aproximación a la cláusula MAC (material adverse change) en los contratos de financiación sindicada a la luz de la jurisprudencia anglo-americana. Navarra, Thomson Reuters Aranzandi, 2016; García Long, Sergio. La estructura contractual de los financiamientos bancarios. En García Long, Sergio (ed). Derecho Contractual Financiero. Estudios en Homenaje a la Facultad de Derecho PUCP en su Centenario. Lima: PUCP, 2019. 
Partiendo de esa premisa, el derecho de contratos es especialmente sensible a la circulación de modelos jurídicos. Un ejemplo concreto de esta circulación es la americanización contractual. Las operaciones corporativas y financieras se basan en un modelo contractual americano distinto al de la ley aplicable, por la simple razón que esta práctica propone cláusulas más adecuadas para las particulares necesidades de una compra de empresas, financiamiento y similares. Por ello, en estos contratos se pactan conditions precedent, reps \& warranties, MAC/MAE, best efforts, reverse termination fees, y otras figuras contractuales que no tiene origen en el Civil Law y que no están reguladas por el Código Civil y que, a pesar de ello, son incluidas en contratos sujetos a ley civilista.

Esta contraposición exige a los abogados corporativos y financieros conocer correctamente el derecho de contratos nacional, luego la figura anglosajona y finalmente concluir si dicha figura anglosajona puede funcionar o no en terreno ajeno civilista, y de funcionar, cómo podría hacerlo. El estudio de los best efforts, HHW y best efforts underwriting pone a prueba lo advertido. 